\title{
Depressão e Desempenho Escolar em Crianças e Adolescentes Institucionalizados
}

\author{
Débora Dalbosco Dell' Aglio ${ }^{12}$ \\ Cláudio Simon Hutz. \\ Universidade Federal do Rio Grande do Sul
}

\begin{abstract}
Resumo
Foram investigadas as variáveis depressão e desempenho escolar em 215 crianças e adolescentes, de ambos os sexos, de 7 a 15 anos, de escolas públicas da periferia de Porto Alegre e Viamão, divididas em 2 grupos. Um grupo de participantes $(n=105)$ estava abrigado em instituições governamentais de proteção especial e o outro $(n=110)$ morava com a familia e freqüentava as mesmas escolas. Os participantes responderam individualmente o Children's Depression Inventory (CDI) e o Raven. As professoras preencheram uma Escala de Avaliação do desempenho dos alunos. Os resultados do CDI indicaram uma média mais alta entre as meninas e no grupo institucionalizado. Foi encontrada uma correlação negativa entre o CDI e o desempenho escolar. As meninas apresentaram uma média mais alta no desempenho escolar e as crianças institucionalizadas uma média mais baixa. Estes resultados indicam a necessidade de estratégias de atendimento específicas para crianças e adolescentes institucionalizados para melhorar seu desempenho escolar e prevenir depressão entre as meninas.

Palavras-chave: Depressão; desempenho escolar; institucionalização.
\end{abstract}

\section{Depression and School Achievement of Institutionalized Children and Adolescents}

\begin{abstract}
The present study investigated depression and school achievement of 215 children and adolescents of both sexes, 7 to 15 years-o ld, who were attending public schools in poor regions of Porto Alegre and Viamão, Brazil. About half the participants $(n=105)$ were living in public institutions. The others $(n=110)$ lived with their families and attended the same schools. The participants completed the Children's Depression Inventory (CDI) and the Raven test. An evaluation scale to assess school achievement was completed by teachers. The CDI scores showed significant differences between the groups. Females living in institutions presented significantly higher scores. A negative correlation was found between the CDI scores and school achievement. Females presented higher school achievement than males but institutionalized children had lower school achievement. These results point to the need of developing specific strategies to deal with institutionalized children and adolescents to improve school performance and to prevent depression, specially among females.

Keywords: Depression; school achievement; institutionalization.
\end{abstract}

Este estudo teve como objetivo investigar a manifestação do distúrbio depressivo e o desempenho escolar em crianças e adolescentes institucionalizados. A depressão é um conceito que tem sido amplamente estudado, tendo em vista a sua alta e crescente prevalência. Em recente revisão sobre a epidemiologia dos transtornos depressivos, em crianças e adolescentes, Bahls (2002) encontrou o resultado da prevalência-ano para a depressão maior de 0,4 a 3,0\% em crianças e de 3,3 a 12,4\% em adolescentes. Na população em geral a prevalência varia em torno de $4 \%$ a 10\% (Bahls \& Bahls, 2002; Zavaschi \& cols., 2002), e também tem sido observada uma maior incidência entre as mulheres, variando de $10 \%$ a $25 \%$, enquanto que entre os homens atinge de $5 \%$ a $12 \%$ (Zavaschi \& cols., 2002). Embora não exista uma definição consensual acerca da depressão, pode-

\footnotetext{
${ }^{1}$ Agradecemos às alunas do Curso de Psicologia da UFRGS, Rosane Zigunovas Zanini e Dinara Bertazo Paz da Silva, e de Priscila Pellin D'Avila da UNISINOS, assim como a bolsista de Aperfeiçoamento Fernanda Ortiz Costa, pela colaboração na coleta, transcrição e categorização dos dados.

${ }^{2}$ Endereço para correspondência: Instituto de Psicologia, Universidade Federal do Rio Grande do Sul, Ramiro Barcelos, 2600, Porto Alegre, RS, 90035 003. Fone: (51)3316-5253, Fax: (51)3330-4797.E-mail:dalbosco@cpovo.net
}

se afirmar que se trata de um distúrbio que sofre a influência de variáveis biológicas, psicológicas e sociais e que se manifesta por meio de sintomas emocionais, como desânimo, baixa autoestima e desinteresse em atividades prazerosas; de natureza cognitiva, como pessimismo e desesperança; motivacionais, como apatia e aborrecimento; e ainda sintomas físicos, tais como perda de apetite, dificuldades para dormir e perda de energia (Compas, Ey \& Grant, 1993; Steinberg, 1999).

Mericangaas e Angst (1995) enumeram alguns fatores de risco e de proteção para o surgimento de depressão ao longo do desenvolvimento. Algumas características do indivíduo e do seu ambiente parecem potencializar os riscos para depressão, como aumento da idade, gênero feminino, baixo nível sócioeconômico, traços de personalidade específicos e presença de fatores ambientais desencadeantes, como perda ou afastamento de seus pais. A presença de história familiar de depressão tem sido considerada um dos mais fortes e potentes fatores de risco para esta desordem. Entre os fatores individuais que parecem proteger os adolescentes da depressão estão: sucesso na vida escolar, envolvimento em atividades extracurriculares, competência social, auto-percepção positiva, competência 
intelectual, relações sociais positivas e suportes sociais adequados. Assim, a maior ou menor probabilidade de surgimento da depressão é vista como o resultado da interação de uma série de condições ambientais, especialmente estresse, perda e predisposições individuais (Steinberg, 1999).

Sobretudo, a falta de apoio familiar, durante a infância e adolescência, tem sido relacionada a manifestações do distúrbio depressivo (Herman-Stahl \& Petersen, 1996; Holahan \& Moos, 1985; Mericangaas \& Angst, 1995). Nesse sentido, diversos estudos apontam para o fato de que vivências traumáticas na infância, como perda de vínculos afetivos devido à morte de pais ou irmãos, ou ainda, a privação de um ou de ambos os pais por separação ou abandono, seriam importantes fatores associados à depressão na vida adulta (Zavaschi \& cols., 2002), embora reconheçam a etiologia das doenças afetivas como de natureza multifatorial. Por outrolado, um contexto familiar que se caracterize por trocas afetivas, intimidade e comunicação apropriada, tem sido identificado como um importante fator de proteção, ajudando as crianças a manterem um senso de estabilidade e rotina frente a mudanças (Herman-Stahl \& Petersen, 1996), mesmo que o relacionamento positivo seja apenas com um dos pais (Ptacek, 1996). Para Steinberg (1999), o adolescente que tem relacionamentos familiares afetuosos e próximos, tem mais condições de enfrentar experiências estressantes do que aqueles sem tal apoio, sendo que esse apoio familiar se constitui no mais importante fator de proteção na adolescência.

Quanto às diferenças de gênero, Rudolph e Hammen (1999) constatam que as adolescentes investem mais do que os adolescentes nos seus relacionamentos, como fonte de apoio emocional e de identidade pessoal, o que as leva, em decorrência, a sentir mais o estresse interpessoal como uma ameaça ao seu próprio bem-estar. Assim, as adolescentes, como por exemplo, em situações de conflitos com os pais e companheiros, experenciam níveis de estresse interpessoal mais altos do que os adolescentes, mostrando-se mais vulneráveis e reagindo mais freqüentemente com respostas depressivas ao estresse. De acordo com Compas e colaboradores (1993), enquanto sentimentos depressivos são mais comuns entre os meninos antes da adolescência, a desordem depressiva é mais comum entre as meninas após a puberdade. Steinberg (1999) também aponta diferenças entre os sexos, demonstrando que há uma maior prevalência de desordens internalizantes, como a depressão, entre as meninas, e desordens externalizantes, como o uso de distração e expressão de sentimentos através de comportamentos agressivos e abuso de drogas ou álcool, entre os meninos. Baron e Campbell (1993) destacam que as garotas relatam mais sintomas subjetivos, como sentimentos de tristeza, vazio, tédio, raiva e ansiedade, e costumam ter, também, mais preocupação com popularidade, menos satisfação com a aparência, e menos auto-estima, enquanto que os garotos relatam mais sentimentos de desprezo, desafio e desdém, e demonstram problemas de conduta como: falta às aulas, fugas de casa, violência física, roubos e abuso de substâncias.

O nível intelectual e o desempenho escolar têm sido considerados como fatores individuais que moderam os efeitos negativos do estresse e se associam a uma menor vulnerabilidade frente ao mesmo (Garmezy, Masten \& Tellegen, 1984). No entanto, o desempenho escolar, dependendo de sua qualidade, pode trazer diferentes conseqüências para a criança: um bom desempenho ajuda a criança a melhorar sua auto-estima, dando-lhe um sentimento de valor pessoal; porém, se os pais ou outros adultos significativos a pressionam exigindo perfeição, esse mesmo desempenho escolar pode também se constituir em fator que torna a criança mais vulnerável. Experiências estressantes ligadas ao ambiente escolar, como aquelas que ocorrem em situações de provas, competições, conflitos com companheiros ou professores, podem levar a resultados não saudáveis, como fobias, queixas somáticas e episódios depressivos (Carson \& Bittner, 1994).

No que se refere aos contextos de desenvolvimento, familia ou instituição, diversos estudos têm relacionado o cuidado institucional a crianças, nos anos iniciais, a dificuldades de comportamento e de personalidade. No entanto, Grusec e Lytton (1988) apontam fatores que podem contribuir na modificação dos efeitos da institucionalização na infância. Entre eles estão as razões para a separação da família, o tipo de relacionamento prévio com a mãe, a oportunidade de desenvolver relações seguras após a separação, a qualidade do cuidado oferecido, a idade da criança, a duração da institucionalização, sexo e temperamento da criança.

Dessa forma, procurando observar o efeito destes diferentes contextos no desenvolvimento, o objetivo específico deste estudo foi verificar a manifestação do distúrbio depressivo e o desempenho escolar em crianças e adolescentes que vivem em instituição de abrigo e em familia.

\section{Método}

\section{Participantes}

Participaram deste estudo 215 crianças e adolescentes, do sexo masculino e do sexo feminino (103 meninos e 112 meninas), com idades entre 7 e 15 anos $(M=10,3$ anos; $d p=1,9)$, que freqüentavam escolas públicas, municipais e estaduais, da periferia das cidades de Porto Alegre e Viamão, e freqüentavam da $1^{\mathrm{a}}$ à $6^{\mathrm{a}}$ série do ensino fundamental, predominando alunos da $2^{\mathrm{a}}$ série. O nível sócio-econômico das familias cujos filhos freqüentam estas escolas tende a ser baixo. Procurou-se compor 2 grupos emparelhados, sendo um de crianças e adolescentes institucionalizados e outro de participantes que moravam com a familia.

Os participantes institucionalizados $(n=105 ; M=10,6$ anos; $d p=1,8)$ estavam abrigados num órgão de proteção especial 
governamental, por motivos de abandono, maus-tratos, negligência, perda dos pais ou decisões judiciais. $\mathrm{O}$ tempo de institucionalização dos participantes da amostra variou de 3 meses a 10 anos $(M=3,6$ anos; $d p=2,5)$. Os participantes deste estudo estavam em abrigos residenciais (até 15 crianças e adolescentes) e em abrigos institucionais (entre 50 a 70 abrigados), sendo que em todos eles os cuidados são dispensados por monitores que se revezam em plantões de atendimento, e são permitidas saídas para escola, passeios e inclusive visitas a familiares.

O grupo de participantes não institucionalizados $(n=110$; $M=9,9$ anos; $d p=1,9)$ foi formado por crianças e adolescentes que estudavam nas mesmas escolas e turmas das crianças institucionalizadas, eque residiam com pelo menos algum membro da sua familia de origem, sendo que $52,7 \%$ dos participantes referiam estar morando com ambos os pais, $29,1 \%$ com apenas um dos pais, $13,7 \%$ com um dos pais e companheiro(a) e 4,5\% com avós, irmãos ou tios.

\section{Instrumentos}

Para investigar o desempenho escolar foi utilizada a Escala de Avaliação (Bandeira \& Hutz, 1994), preenchida pelas professoras, que avalia o desenvolvimento da aprendizagem em sala de aula, concentração nas tarefas, relacionamento com colegas e professores e desempenho em tarefas específicas, como escrita, leitura e matemática, entre outras. Este instrumento mostrou-se consistente em estudos anteriores (alpha de Cronbach $=0,93$ ), tendo sido demonstrado que os professores são capazes de utilizá-la para avaliar alunos objetivamente (Giacomoni, 1998; Hutz \& Bandeira, 1995). Esta escala é composta por 33 itens, do tipo Likert com 5 pontos, que vão de concordo plenamente a discordo plenamente, com uma amplitude de 33 a 165.

Para medir depressão foi utilizado o Children's Depression Imventory (CDI) (Kovacs, 1992). O CDI foi elaborado por Kovacs, adaptado do Beck. Depression Inventory para adultos. O objetivo do CDI é detectar a presença e a severidade do transtorno depressivo na infância. Destina-se a identificar alterações afetivas em crianças e adolescentes dos 7 aos 17 anos de idade. Este inventário é composto por 27 itens, cada um com três opções de resposta. A criança deve escolher a opção que melhor descreve o seu estado nos últimos tempos. As opções são pontuadas de $0 \mathrm{a} 2 \mathrm{e}$ o teste pode ser aplicado individual ou coletivamente. A consistência interna descrita por Kovacs mostrou-se adequada $(0,86)$, e o ponto de corte do CDI foi estabelecido em 19 pontos. O CDI já foi adaptado, para uso em João Pessoa, por Gouveia, Barbosa, Almeida e Gaião (1995) e vem demonstrando características psicométricas adequadas. Este instrumento também foi utilizado em pesquisas no Rio Grande do Sul (Giacomoni, 1998) apresentando um alpha de Cronbach igual a 0,82 e uma correlação negativa com nível de satisfação de vida $(r=-0,25)$.

Para controlar possíveis efeitos do nível de inteligência sobre as variáveis de interesse também foi utilizado o Teste das Matrizes
Progressivas Coloridas de Raven (Escala Especial), que é um teste de inteligência adaptado para o uso em crianças. Este teste foi normatizado para crianças brasileiras por Angelini, Alves, Custódio, Duarte e Duarte (1999) e é apropriado para avaliar crianças na faixa etária de 5 a 11 anos, pessoas portadoras de deficiência mental e pessoas idosas. O teste contém três séries: $\mathrm{A}, \mathrm{Ab}$ e B, cada uma com 12 problemas.

\section{Procedimentos}

A composição da amostra partiu de uma listagem, fornecida pela Instituição de abrigo governamental, indicando a escola que as crianças e os adolescentes estudavam. Foi, então, obtida uma lista de participantes que freqüentavam as mesmas turmas do grupo institucionalizado, tivessem o mesmo sexo, morassem com algum membro de sua familia original e tivessem a data de nascimento mais próxima possível da criança correspondente.

A aplicação dos instrumentos foi realizada nas escolas públicas, estaduais e municipais, nas quais os participantes estudavam. Cada criança ou adolescente foi testado individualmente, em sala apropriada. O CDI foi aplicado de forma oral, ou seja, as questões foram lidas para todos os participantes por que muitos deles apresentavam dificuldades de leitura. A Escala de Avaliação do desempenho dos alunos foi preenchida pela professora titular nas turmas de $1^{\mathrm{a}}$ a $4^{\mathrm{a}}$ série do ensino fundamental, ou pela professora "regente" nas turmas de $5^{\mathrm{a}}$ e $6^{\mathrm{a}}$ série.

\section{Considerações Éticas}

Foram tomados cuidados éticos apropriados ao tipo de população investigada neste estudo. Esta pesquisa foi classificada como de risco mínimo para as crianças e adolescentes. Foi obtido Consentimento Informado da Instituição governamental que mantém formalmente a guarda das crianças e adolescentes abrigados, assim como da direção das escolas participantes, conforme orientações éticas para pesquisas com seres humanos (Hutz \& Silva, 2002; Hutz \& Spink, 2000; Lisboa \& Koller, 2000). Também foi solicitada a cada participante a concordância em participar da pesquisa, assegurando-lhes sigilo e confidencialidade dos dados.

\section{Resultados}

\section{Características Psicométricas dos Instrumentos Utilizados}

A Escala de Avaliação apresentou uma média de 106,8 $(d p=26,8)$ e uma consistência interna elevada (alpha de Cronbach $=0,94)$, demonstrando resultados equivalentes aos obtidos nos estudos anteriores (Giacomoni, 1998).

O CDI, instrumento utilizado para avaliar a depressão, apresentou uma consistência interna de 0,79 , medida através do alpha de Cronbach. A média encontrada na escala foi de 14,6 $(\phi p=7,1)$. 
No Teste de Matrizes Coloridas de Raven utilizou-se os escores brutos finais do teste, encontrados pela soma das séries A, Ab e B de cada particpante. A média geral foi 20,5 $(d p=5,9)$. Foi encontrada uma diferença significativa no Raven, entre o grupo institucionalizado $(M=19,8)$ e o grupo que mora com a família $(M=21,1)$, quando se covariou o efeito da idade $[F(1,212)=5,1 ; p<0,02]$. Todavia, embora a diferença seja significativa, ela é de pouca relevância prática, pois o tamanho do efeito é pequeno $(d=0,21)$ (Cohen, 1988). A diferença observada equivale, numa escala usual de QI, como a escala WISC $(M=100 ; d p=15)$, a uma diferença de aproximadamente 3 pontos. Não foram encontradas outras diferenças significativas.

\section{Depressão}

Foi realizada uma ANOVA 2×2x2 (sexo, moradia e faixa etária) com os resultados do CDI, covariando os escores do Raven. Foram encontradas diferenças significativas entre os sexos $[F(1$, 204) $=5,45 ; p<0,021]$ e entre o grupo institucionalizado e o grupo não institucionalizado $[F(1,204)=6,0 ; p<0,015]$, indicando que as meninas $(M=15,8)$ apresentaram uma média mais alta do que os meninos ( $M=13,0)$, e que o grupo institucionalizado $(M=15,8)$ apresentou uma média mais alta do que o grupo que mora com a familia $(M=13,5)$. As interações não foram significativas. Os resultados referentes ao CDI, por sexo, moradia e faixa etária, são apresentados na Tabela 1.

Em estudos epidemiológicos com o CDI, considera-se 2 desvios-padrão acima da média como ponto de corte para provável diagnóstico de depressão (Gouveia \& cols., 1995). Utilizando esse critério (um escore de 29 pontos ou superior no nosso estudo), foram identificados 13 participantes ( $6 \%$ da amostra) com provável diagnóstico de depressão.

\section{Escala de Avaliação - Desempenho Escolar}

Foi realizada uma ANOVA 2x2x2 (sexo, moradia e faixa etária) com os resultados da Escala de Avaliação, covariando os escores do Raven. Essa análise mostrou que houve uma interação significativa entre faixa etária e moradia $[F(1,202)=7,9 ; p<0,005]$. Foi então realizado um Teste $t$ comparando os resultados dos participantes institucionalizados e os que moravam com a familia, para cada faixa etária. Constatou-se que houve diferença significativa entre os escores da Escala de Avaliação no grupo de crianças de $7-10$ anos $(t=3,03 ; g l=112 ; p<0,003)$, sendo que as crianças da instituição apresentaram uma média mais baixa ( $M=98,7, d p=21,31)$ do que as crianças que moram com a familia $(M=113,82, \not p=29,04)$. Entre os adolescentes (11-15 anos) não foi encontrada diferença significativa. Também foi encontrada uma diferença significativa entre os $\operatorname{sexos}[F(1,202)=9,8 ; p<0,002]$,

Tabela 1

Resultados do CDI por Situação de Moradia, Sexo e Faixa Etária

\begin{tabular}{lllllllll}
\hline Variável & & $n$ & $M$ & $d p$ & $E P M$. & L.I. & L.S. & Raven \\
\hline Situação de Moradia & Instituição & 104 & 15,8 & 8,0 & 0,66 & 14,5 & 17,1 & 19,8 \\
& Família & 109 & 13,5 & 5,9 & 0,66 & 12,2 & 14,8 & 21,1 \\
Sexo & Meninos & 101 & 13,6 & 6,8 & 0,67 & 12,3 & 14,9 & 21,1 \\
\multirow{2}{*}{ Faixa Etária } & Meninas & 112 & 15,8 & 7,3 & 0,64 & 14,5 & 17,0 & 19,9 \\
& $7-10$ anos & 116 & 15,1 & 7,2 & 0,63 & 13,8 & 16,3 & 19,3 \\
& $11-15$ anos & 97 & 14,3 & 7,0 & 0,69 & 12,9 & 15,6 & 21,8
\end{tabular}

Nota. EPM= erro-padrão da média; $d p=$ desvio- padrão; L.I.= limite inferior do intervalo de confiança; L.S.= limite superior do intervalo de confiança.

Tabela 2

Resultados na Escala de Avaliação por Sexo, Moradia e Faixa Etária

\begin{tabular}{llllllll}
\hline Variáveis & & $n$ & $M$ & $d p$ & $E P M$ & L.I. & L.S. \\
\hline Situação de Moradia & Instituição & 104 & 104,4 & 25,9 & 2,4 & 99,5 & 109,2 \\
& Família & 107 & 106,3 & 27,5 & 2,5 & 101,4 & 111,2 \\
Sexo & Meninos & 99 & 99,9 & 26,9 & 2,5 & 94,9 & 104,8 \\
& Meninas & 112 & 110,8 & 25,9 & 2,4 & 106,1 & 115,5 \\
Faixa Etária & $7-10$ anos & 114 & 107,6 & 27,1 & 2,4 & 102,9 & 112,3 \\
& $11-15$ anos & 97 & 103,0 & 26,6 & 2,6 & 97,9 & 108,1 \\
\hline
\end{tabular}

Nota. $E P M=$ erro-padrão da média; $d p=$ desvio- padrão; L.I.= limite inferior do intervalo de confiança; L.S.= limite superior do intervalo de confiança. 
Tabela 3

Correlacões entre Variáveis

\begin{tabular}{llcclcll}
\hline & Idade & Sexo & $\begin{array}{c}\text { Série } \\
\text { Escolar }\end{array}$ & $\begin{array}{c}\text { Tempo na } \\
\text { Instituição }\end{array}$ & $\begin{array}{c}\text { Situação } \\
\text { Moradia }\end{array}$ & $\begin{array}{c}\text { Raven } \\
\text { Escala de } \\
\text { Avaliação }\end{array}$ \\
\hline Sexo & 0,05 & - & & & & & \\
Série Escolar & $0,40^{* *}$ & $-0,03$ & - & & & & \\
Tempo na Instituição & 0,09 & $-0,05$ & $0,39^{* *}$ & - & & & \\
Situação de Moradia & $-0,18^{* *}$ & 0,01 & 0,11 & - & - & & \\
Raven & $0,22^{* *}$ & $-0,10$ & $0,57^{* *}$ & $0,30^{* *}$ & 0,10 & - & \\
Escala de Avaliação & $-0,02$ & $0,18^{* *}$ & $0,20^{* *}$ & $-0,06$ & 0,10 & $0,29^{* *}$ & - \\
CDI & $-0,06$ & $0,18^{* *}$ & $-0,29^{* *}$ & $-0,09$ & $-0,18^{* *}$ & $-0,28^{* *}$ & $-0,24^{* *}$ \\
\hline
\end{tabular}

Nota. $* * p<0,01$

sendo que as meninas $(M=110,8)$ apresentaram uma média mais alta do que os meninos $(M=99,9)$. Os resultados referentes à Escala de Avaliação, por sexo, moradia e faixa etária, são apresentados na Tabela 2.

\section{Correlações}

Na Tabela 3 são apresentadas as correlações, obtidas através do teste de Pearson, entre idade, sexo, série escolar, tempo de institucionalização, situação de moradia, escores no Raven, Escala de Avaliação e CDI.

A Escala de Avaliação apresentou correlações significativas com sexo $(r=0,18)$, indicando melhor desempenho das meninas; série $(r=0,20)$, Raven $(r=0,29)$ e com o CDI $(r=-0,24)$.

O CDI apresentou correlações significativas com sexo $(r=0,18)$,indicando maioresíndices de depressão entre as meninas; série $(r=-0,29)$; Raven ( $r=-0,28)$; Escala de Avaliação $(r=-0,24)$; e moradia $(r=-0,18)$, indicando que há mais depressão no grupo de crianças e adolescentes institucionalizados.

Também foram encontradas correlações significativas entre o Raven e idade $(r=0,22)$; série $(r=0,57)$; Escala de Avaliação $(r=0,29)$; CDI $(r=-0,28)$; e tempo de permanência na instituição $(r=0,30)$. Exceto pela última correlação, as demais eram esperadas.

\section{Discussão}

Os resultados deste estudo possibilitaram a avaliação do desempenho escolar e da presença de distúrbio depressivo entre as crianças e adolescentes institucionalizados e não institucionalizados, controlando nível intelectual. O instrumento utilizado para avaliar o desempenho escolar do aluno, a Escala de Avaliação, mostrou-se consistente, apresentando correlações importantes com a série freqüentada e com os resultados do Raven, indicando que é adequado para avaliar o que se propõe.

Quanto ao desempenho escolar, foi encontrada diferença entre o grupo institucionalizado e o grupo que mora com a família apenas entre as crianças, sendo que o grupo institucionalizado apresentou médias mais baixas. Este resultado confirma a idéia inicial de que a familia desempenha um papel importante no desempenho escolar das crianças. Não houve diferença significativa entre os adolescentes, indicando que nesta faixa etária as diferentes redes de apoio disponíveis não parecem produzir efeitos na avaliação que é realizada pelas professoras. Esse achado sugere que a falta de apoio familiar pode repercutir mais no desempenho escolar das crianças do que dos adolescentes. Isso ocorre, provavelmente, porque este é um período em que há uma maior dependência das crianças em relação aos adultos e é necessária a presença de um ambiente organizado, com afeto e autoridade, além de uma expectativa positiva em relação às crianças (Hardy, Power \& Jaedicke, 1993). Geralmente, essas condições são mais facilmente encontradas em uma familia do que em uma instituição. Durante a adolescência, além da família, outros fatores contribuem muito para o processo de desenvolvimento. $\mathrm{O}$ acesso a outros recursos sociais, como trabalho, e as relações com pares podem afetar significativamente o desempenho escolar de adolescentes.

O Teste das Matrizes Progressivas Coloridas de Raven apresentou diferença significativa entre o grupo institucionalizado e o grupo que mora com a familia, com uma média mais alta entre as crianças que moram com a familia. No entanto esta diferença é pequena e não tem significado clínico. Na prática, $\mathrm{O}$ nível intelectual dos dois grupos é similar. A correlação positiva encontrada entre o tempo de permanência na instituição e o resultado do Raven é mais um dado que, de certa forma, pode estar indicando que a instituição também colabora no desenvolvimento intelectual das crianças que lá permanecem ou, de alguma forma, provê atividades que auxiliam estas crianças e adolescentes a se organizarem e desenvolverem tarefas cognitivas, podendo se constituir num fator positivo para o desenvolvimento.

Quanto aos resultados encontrados no Children's Depression Inventory (CDI), o índice de $6 \%$ do total da amostra com escores significativos para depressão é semelhante aos índices apontados 
pela literatura (Bahls, 2002; Barbosa, Dias, Gaião \& Di Lorenzo, 1996), que variam entre $4 \%$ a $10 \%$ em crianças e adolescentes. De qualquer forma, é importante destacar, que a definição do ponto de corte para depressão não deve seguir apenas um critério psicométrico, mas exige também uma avaliação clínica, que possibilite a comprovação da manifestação do distúrbio depressivo através de critérios diagnósticos definidos e permita estimar a precisão do instrumento.

A diferença entre os sexos encontrada no CDI, indicando escores mais altos entre as meninas, confirma os resultados de estudos como os de Compas e colaboradores (1993) e Barbosa e colaboradores (1996). Estes dados também podem estar refletindo algo que ocorre freqüentemente nesta população que é a maior freqüência de violência doméstica, abuso sexual e negligência contra meninas (Kristensen, Oliveira \& Flores, 2000; Steinberg, 1999). Além disso, Mericangaas e Angst (1995) e Steinberg (1999) apontam a perda e separação dos pais como um fator de risco para o surgimento de depressão, que pode ser potencializado pela variável sexo, já que a depressão se mostra mais freqüente no grupo feminino.

Também foi observada uma diferença significativa nos escores do CDI, entre o grupo institucionalizado e o grupo que mora com a familia, apontando uma maior depressão entre as crianças e adolescentes institucionalizados, corroborando estudos que apontam a falta de apoio familiar como um preditor para depressão (Mericangaas \& Angst, 1995; Steinberg, 1999). Pode-se entender que, embora as instituições em geral sejam consideradas "boas", na medida em que são vistas como um órgão provedor, supridor das necessidades básicas de segurança e proteção contra o mundo externo, continua existindo uma lacuna no que se refere aos vínculos afetivos básicos que de alguma forma foram rompidos ou não se constituíram. Existem referências apontando que crianças que sofreram rompimentos bruscos com seus vínculos anteriores, mesmo que perturbados, sofrem seqüelas sociais e emocionais, oriundas da disfunção do apego criada em sua dinâmica familiar, como atitudes defensivas contra um ambiente inseguro e ameaçador, desconfiança básica, agressividade, sentimentos de culpa, baixa auto-estima e conduta autodestrutiva (Loos, Ferreira \& Vasconcelos, 1999).

Destaca-se, no entanto, que os abrigos de proteção, onde estão os participantes deste estudo, não se caracterizam como "Instituições Totais", conforme refere Goffman (1961), na medida em que os mesmos não se mantêm separados da sociedade mais ampla, funcionam em regime aberto, permitindo a saída das crianças e adolescentes para atividades na comunidade. Para Altoé (1990), em geral, abrigos de proteção ou "internatos", apresentam um atendimento mais impessoal e despersonalizado, dificultando a construção de laços afetivos e significativos, levando à pobreza nos relacionamentos interpessoais. Também refere que a instituição deixa marcas no indivíduo que passa ali anos de sua infância e adolescência, marcas que influenciam sua trajetória e sua inserção na vida social, podendo dificultar seu desenvolvimento psicológico, da inteligência e criatividade (Altoé, 1993). Além disso, a criança institucionalizada geralmente tem uma visão negativa de si mesmo, restringindo, dessa forma, as relações de aceitação social que são reforçadas pelo estigma institucional, que conforme Bronfenbrenner (1979/1996) pode se tornar uma profecia de fracasso na vida destas crianças. Podese considerar que, mesmo que a instituição ofereça o atendimento de necessidades básicas ao desenvolvimento de crianças e adolescentes, ela não oferece condições para um atendimento individualizado, com estabelecimento de laços afetivos, que podem ser alcançados mais facilmente num ambiente familiar. No entanto, essa questão é muito complexa e seriam necessários novos estudos para compreender os efeitos da institucionalização ao longo do desenvolvimento.

A depressão, avaliada através do CDI, também apresentou correlações negativas com os resultados do Raven e da Escala de Avaliação, especialmente entre as crianças com índices de depressão mais severos. A literatura em geral tem apontado correlações entre depressão, baixo nível intelectual e baixo desempenho escolar. Em pesquisa sobre o rendimento escolar em crianças de 9 a 12 anos com sintomas depressivos, de uma escola particular na cidade do Recife, Bandim, Roazzi e Doménech (1998) obtiveram como resultado um prejuízo significativo no desempenho escolar em todas as matérias, principalmente em Português e Ciências, quando comparados com crianças sem sintomas depressivos. No entanto, neste estudo, não foi possível apontar de forma clara qual a direção da relação. A criança pode não ter um bom desempenho na escola e nos testes de inteligência porque está deprimida, com baixa auto-estima e com sentimentos de baixa auto-eficácia. Também é possível que, por ter menos condições intelectuais e menor desempenho acadêmico, apresente índices maiores de depressão porque não se sente capaz de lidar com as demandas impostas pelo meio. Esta é uma questão que precisa ser mais investigada para que se possa pensar em estratégias de atendimento eficazes e intervenções precoces para crianças e adolescentes que apresentam a síndrome depressiva. Também se torna necessária eimportante a discussão de ações diferenciadas e de estudos específicos para melhorar a qualidade de vida desta população, especialmente das meninas institucionalizadas que apresentaram índices mais elevados de depressão.

Finalmente, os resultados encontrados apontam para a necessidade de investigações sobre a incidência dos diferentes tipos de estressores e seus efeitos no bem-estar psicológico de crianças e adolescentes, assim como sobre os fatores de risco associados à síndrome depressiva. 


\section{Referências}

Altoé, S. (1990). Infancias perdidas: O cotidiano nos internatos-prisão. Rio de Janeiro: Xenon.

Altoé, S. (1993). Menores em tempo de maioridade: Do internato-prisão à vida social Rio de Janeiro: Santa Úrsula.

Andriola, W. B. \& Cavalcante, L. R. (1999). Avaliação da depressão infantil em alunos da pré-escola. Psicologia: Reflexão e Crítica, 12, 419-428.

Angelini, A. L., Alves, I. C. B., Custódio, E. M., Duarte, W. F. \& Duarte, J. L. M. (1999). Manual de matrizes progressivas de Raven-Escala especial. São Paulo: Centro Editor de Testes e Pesquisas em Psicologia.

Bahls, S. C. (2002). Epidemiology of depressive symptoms in adolescents of a public school in Curitiba, Brazil. Revista Brasileira de Psiquiatria, 24, 63-67.

Bahls, S. C. \& Bahls, F. R. C. (2002). Depressão na adolescência: Características clínicas. Interação em Psicologia, 6, 49-57.

Bandeira, D. R. \& Hutz, C. S. (1994). A contribuição dos Testes DFH, Bender e Raven na predição do rendimento escolar na primeira série. Psicologia: Teoria e Pesquisa, 10, 59-72.

Bandim, J. M., Roazzi, A. \& Doménech, E. (1998). Rendimento escolar em crianças com sintomas depressivos. Jornal Brasileiro de Psiquiatria, 47, 353-460.

Barbosa, G. A., Dias, M. R., Gaião, A. A. \& Di Lorenzo, W. F. (1996). Depressão infantil: Um estudo de prevalência com o CDI. Infanto, 3, 36-40.

Baron, P. \& Campbell, T. L. (1993). Gender differences in the expression of depressive symptoms in middle adolescents: An extension of early findings. Adolescence, $28,903-911$

Bronfenbrenner, U. (1996). A ecologia do desenvolvimento bumano: Experimentos naturais eplanejados. Porto Alegre: Artes Médicas. (Original publicado em 1979)

Carson, D. K. \& Bittner, M. T. (1994). Temperament and school-aged children's coping abilities and responses to stress. The Journal of Genetic Psychology, 155, 289-302.

Cohen, J. (1988). Statistical power analysisfor the behavioral sciences. Hillsdale: Lawrence Erlbaum.

Compas, B. E., Ey, S. \& Grant, K. (1993). Taxonomy, assessment, and diagnosis of depression during adolescence. Psychological Bulletin, 114, 323-344.

Garmezy, N., Masten, A. S. \& Tellegen, A. (1984). The study of stress and competence in children: A building block for developmental psychopathology. Child Development, 55, 97-111.

Giacomoni, C. H. (1998). Desempenho acadêmico, controle percebido e eventos de vida como preditores de bem-estar subjetivo em crianças. Dissertação de Mestrado não-publicada, Curso de Pós-Graduação em Psicologia do Desenvolvimento, Universidade Federal do Rio Grande do Sul. Porto Alegre, RS.

Goffman, E. (1961). Manicômios, prisões e conventos. São Paulo: Perspectiva.

Gouveia, V. V., Barbosa, G. A., Almeida, H. J. F. \& Gaião, A. A. (1995). Inventário de depressão infantil- CDI: Estudo de adaptação com escolares de João Pessoa. Jornal Brasileiro de Psiquiatria, 44, 345-349.

Grusec, J. \& Lytton, H. (1988). Social development: History, theory and research. New York: Springer-Verlag.
Hardy, D. F., Power, T. G. \& Jaedicke, S. (1993). Examining the relation of parenting to children's coping with everyday stress. Child Development, 64, 1829-1841.

Herman-Stahl, M. \& Petersen, A. C. (1996). The protective role of coping and social resources for depressive symptoms among young adolescents. Journal of Youth and Adolescence, 25, 733-753.

Holahan, C. J. \& Moos, R. H. (1985). Life stress and health: Personality, coping, and family support in stress resistance. Journal of Personality and Social Psychology, 49, 739-747.

Hutz, C. S. \& Bandeira, D. R. (1995). Avaliação psicológica com o desenho da figura humana: Técnica ou intuição? Temas em Psicologia, 3, 35-41.

Hutz, C. S. \& Silva, D. F. (2002). Avaliação psicológica de crianças em situação de risco. Avaliação Psicológica, 1, 73-79.

Hutz, C. S. \& Spink, M. J. (2000). Orientaçoes éticas para psicólogos envolvidos em pesquisa com seres humanos. Documento elaborado para o Fórum das Entidades Nacionais da Psicologia. Retirado de: wnw.psicologia.ufrgs.br/laboratorio em 10/10/2003.

Kovacs, M. (1992). Children's depression inventory manual. Los Angeles: Western Psychological Services.

Kristensen, C. H., Oliveira, M. S. \& Flores, R. Z. (2000). Violência contra crianças e adolescentes na Grande Porto Alegre. Parte B: Pode piorar? Em AMENCAR (Org.), Violência doméstica (pp. 104-117). Brasília: UNICEF.

Lisboa, C. S. M. \& Koller, S. H. (2000). Questões éticas na pesquisa com crianças e adolescentes vítimas de violência doméstica. Alethéia, 11, 59-70.

Loos, H., Ferreira, S. P. A. \& Vasconcelos, F. C. (1999). Julgamento moral: Estudo comparativo entre crianças institucionalizadas e crianças da comunidade de baixa renda com relação à emergência do sentimento de culpa. Psicologia: Reflexão e Crítica, 12, 47-69.

Mericangaas, K. R. \& Angst, J. (1995). The challenge of depressive disorders in adolescence. Em M. Rutter (Org.), Psychosocial disturbances in young people (pp. 36). Londres: Cambridge University Press.

Ptacek, J. T. (1996). The hole of attachment in perceived support and the stress and coping process. Em G. R. Pierce, B. R. Sarason \& I. G. Sarason (Orgs.), Handbook of social support and the family (pp. 495-520). New York: Plenum Press.

Rudolph, K. D. \& Hammen, C. (1999). Age and gender as determinants of stress exposure, generation, and reactions in youngsters: A transactional perspective. Child Development, 70, 660-677.

Steinberg, L. (1999). Adolescence. Boston: McGraw-Hill.

Zavaschi, M. L. S, Satler, F., Poester, D. Vargas, C. F., Piazenski, R., Rohde, L. A. P. \& Eizirik, C. L. (2002). Associação entre trauma por perda na infância e depressão na vida adulta. Revista Brasileira de Psiquiatria, 24, 189-195.

Sobre os autores

Débora Dalbosco Dell'Aglio é Professora do Programa da Pós-graduação em Psicologia do Desenvolvimento da Universidade Federal do Rio Grande do Sul.

Cláudio Simon Hutz é Professor do Programa da Pós-graduação em Psicologia do Desenvolvimento da Universidade Federal do Rio Grande do Sul. 